\title{
Das Arztzeugnis - Teil 2
}

Iris Herzog-Zwitter ${ }^{a}$, Bruno Soltermann ${ }^{b}$, Andreas Klipstein ${ }^{c}$, Gerhard Ebner

${ }^{a}$ Dr. iur., Juristin, FMH Rechtsdienst, Bildungsbeauftragte Swiss Insurance Medicine (SIM); ${ }^{b}$ Dr. med., MAS Versicherungsmedizin, Vorsitzender Weiter- und Fortbildungskommission der SIM, Chefarzt Schweizerischer Versicherungsverband; ${ }^{C}$ PD Dr. med. MSc, Leiter Medizin AEH AG, Fachleiter ZAFAS Kurse SIM; ${ }^{d}$ Dr. med. M.H.A., Präsident SIM, Facharzt für Psychiatrie und Psychotherapie, Praxisinhaber, Zürich

Dies ist der zweite Teil einer kleinen Reihe von Beiträgen, welche die vielfältigen Facetten von Arbeitszeugnissen im Spannungsfeld von Medizin und Recht erörtern und Hinweise für den korrekten Umgang geben. Das gerade auch vor dem Hintergrund neuer Aspekte wie etwa Telemedizin.

\section{Arztzeugnis und FMH-Standesordnung}

Ein nicht lege artis ausgestelltes Arztzeugnis kann standesrechtliche Konsequenzen nach sich ziehen. Art. 34 der FMH-Standesordnung definiert die Anforderungen an ein Arztzeugnis mit folgendem Wortlaut: "Ärztliche Zeugnisse, Berichte und Gutachten sind Urkunden. Bei deren Ausstellung haben Arzt und Ärztin alle Sorgfalt anzuwenden und nach bestem Wissen ihre ärztliche Überzeugung auszudrücken. Der Zweck der Schriftstücke, das Ausstellungsdatum und ihre Empfänger sind anzugeben. Die Ausstellung von Gefälligkeitszeugnissen ist unzulässig.»
Das Arztzeugnis muss die ärztliche Beurteilung eindeutig wiedergeben. Das Bundesgericht definiert die Anforderungen an den Arzt wie folgt: «Bei der Beurteilung, ob das Gesundheitszeugnis mit der Wahrheit übereinstimmt, ist zu berücksichtigen, dass es auf einem Sachverhalt beruht, der durch den Arzt interpretiert wird, und damit gezwungenermassen eine subjektive Komponente enthält» [1].

Das Arztzeugnis hat sich auf die notwendigen Informationen für den Empfänger zu beschränken, weitere Informationen wie beispielsweise eine Diagnose oder Behandlungen dürfen nur mit Einwilligung des Patienten dem Arbeitgeber mitgeteilt werden. In einem Arbeitsunfähigkeitszeugnis ist die differenzierte Arbeits-

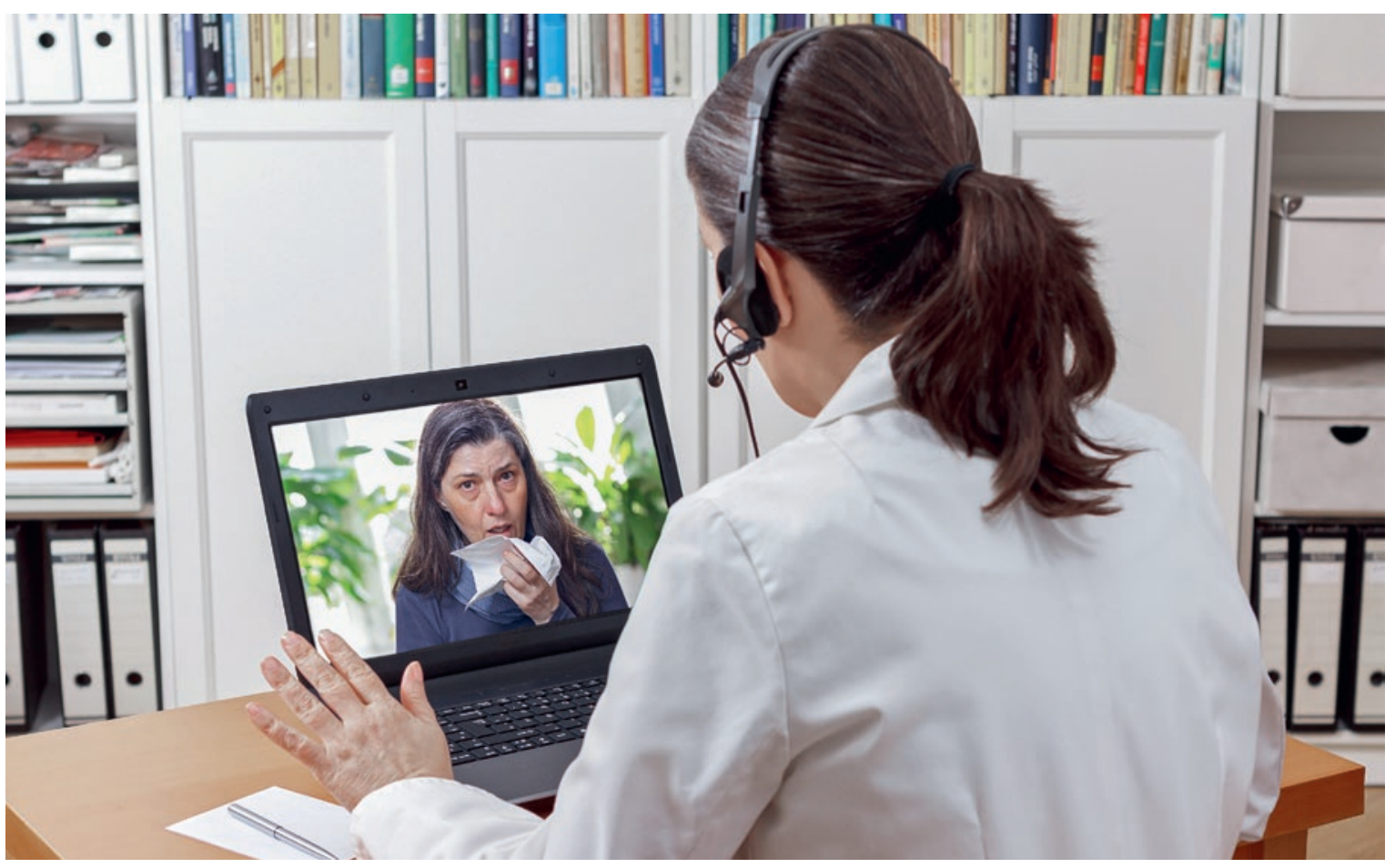

Welchen Beweiswert hat ein Arztzeugnis, das auf einer telemedizinischen Konsultation basiert? 
fähigkeitsbeurteilung darzulegen, zu begründen und durch den Grad der Arbeitsunfähigkeit auszudrücken. Die Administration des Versicherers oder die Richter entscheiden, ob eine Krankheit, ein Unfall, eine Berufskrankheit oder eine unfallähnliche Körperschädigung gegeben ist.

\section{Arztzeugnis nach erfolgter Telekonsultation}

Telemedizin hat nicht nur zunehmend durch die Covid-19-Pandemie ihren Platz im medizinischen Alltag eingenommen, auch im Rahmen des Massnahmenpakets Kostendämpfung 2 wird sie auf Gesetzgebungsebene erwähnt [2]. Der Gesetzgeber sieht unter

\section{Eine eingescannte Unterschrift erfüllt die rechtliche Verbindlichkeit einer Urkunde nicht.}

anderem vor, Erstberatungsstellen als telemedizinische Zentren einzurichten [3].

Dass per telemedizinische Konsultation ein Arztzeugnis ausgestellt werden kann, ist unbestritten. Aber es stellt sich die Frage, welchen Beweiswert ein Arztzeugnis basierend auf einer telemedizinischen Konsultation hat. Auf jeden Fall ist davon auszugehen, dass die Beweiskraft dieser Urkunde geringer sein wird als diejenige des herkömmlichen Arztzeugnisses, da dieses basierend auf "sorgfältigen und fachkundigen Untersuchungen der Ärztin bzw. des Arztes erstellt» wird mit dem persönlichen Kontakt zur Patientin [4].

Medgate, ein Anbieter von telemedizinischen Leistungen, weist in ihren Richtlinien darauf hin, dass per Telefon oder Video eine Teil-Arbeitsunfähigkeit nicht beurteilt wird. Deshalb bescheinigt Medgate eine Arbeitsunfähigkeit nur im Umfang von 100\% und hält fest, dass lediglich die Ausstellung eines einfachen Arztzeugnisses möglich ist [5].

Interessant ist ein aktuelles telemedizinisches Pilotprojekt für Covid-19-Patienten im Tessin [6]. Die Frage der Beweiskraft von telemedizinischen Arbeitsunfähigkeitsbescheinigungen wird in Zukunft vermehrt die Gerichte befassen.

\section{Arztzeugnis - elektronisch signiert}

Grundsätzlich muss das Arztzeugnis entweder eigenhändig durch den Arzt unterschrieben werden oder es benötigt eine qualifizierte elektronische Unterschrift nach ZertES (Bundesgesetz über die elektronische Signatur) [7]. Letztere muss also auf elektronisch übermittelten Arztzeugnissen vorhanden sein.
Eine eingescannte Unterschrift oder der Hinweis des Arztes, das Dokument sei elektronisch visiert worden, erfüllt die rechtliche Verbindlichkeit einer Urkunde nicht. Zudem erfüllt weder ein E-Mail noch eine Fotokopie die Anforderungen an eine Urkunde.

\section{Vertrauensarzt untersteht gegenüber dem Arbeitgeber dem Berufsgeheimnis}

Das Bundesgericht hat in einem Leiturteil BGE 143 IV 209 präzisiert, inwieweit der Vertrauensarzt gegenüber dem Arbeitgeber dem Berufsgeheimnis untersteht [8]. Das Bundesgericht bestätigt, dass der Vertrauensarzt dem Arbeitgeber nur so weit Auskunft erteilen kann, als er vom Arztgeheimnis befreit ist. Das Bundesgericht spricht in diesem Leiturteil vom «Vertrauensarzt», was jedoch nicht dem im Art. 57 KVG erwähnten Vertrauensarzt entspricht. Dieser wird gemäss Gesetzgeber als unabhängige Instanz zur Wirtschaftlichkeits- und Qualitätskontrolle eingesetzt.

Das Bundesgericht führt dazu wie folgt aus: «Sowohl der Arzt, der vom Patienten selber aufgesucht wird, als auch der Vertrauensarzt, der von einem Arbeitgeber eingesetzt wird, werden wegen ihrer berufsspezifischen Kenntnisse und Fähigkeiten in Anspruch genommen. Auch der vom Arbeitgeber eingesetzte Vertrauensarzt muss über umfassende Informationen über den Gesundheitszustand des Exploranden verfügen, um der ihm übertragenen Aufgabe sachgerecht nachkommen zu können. Der Arbeitnehmer, der zu

\section{Das Bundesgericht bestätigt, dass der Vertrau-} ensarzt nur so weit Auskunft erteilen kann, als er vom Arztgeheimnis befreit ist.

einer vertrauensärztlichen Untersuchung aufgeboten wird, darf darauf vertrauen, dass diese Informationen nicht ohne Weiteres an den Arbeitgeber weitergeleitet werden» [9].

Ob und in welchem Umfang der Vertrauensarzt dem Arbeitgeber berichten darf, hängt davon ab, ob er seitens des Arbeitnehmers vom Geheimnis entbunden worden ist. Ohne weitergehende Ermächtigung des Arbeitnehmers darf sich der Vertrauensarzt gegenüber dem Arbeitgeber nur zum Bestehen, zur Dauer und zum Grad einer Arbeitsfähigkeit äussern sowie zur Frage, ob es sich um eine Krankheit oder einen Unfall handelt. Erteilt der Patient dem Arzt die Ermächtigung für eine Arbeitsfähigkeitsbeurteilung zuhanden des Arbeitgebers, so ist der Arzt basierend auf der Ermächtigung legitimiert, beim Arbeitgeber diejenigen Informationen einzuholen, die für die Beurteilung der Arbeitsunfähigkeit bzw. Arbeitsfähigkeit notwendig sind. 
Eine Person, die ein Geheimnis mit der Einwilligung des Berechtigten offenbart, ist gemäss Art. 322 Ziff. 2 StGB nicht strafbar. Der Arbeitgeber darf vom Vertrauensarzt zudem gemäss Art. 328b OR nur diejenigen Angaben erfragen, welche die Eignung des Arbeitnehmers für das Arbeitsverhältnis betreffen oder zur Durchführung des Arbeitsverhältnisses erforderlich sind. Eine Diagnose darf nicht erfragt werden.

\section{Strafrechtliche Aspekte im Zusammen- hang mit der Ausstellung eines Arzt- zeugnisses}

Stellt eine Ärztin vorsätzlich oder fahrlässig ein falsches Zeugnis aus, dann ist der Straftatbestand des falschen ärztlichen Zeugnisses erfüllt. In Art. 34 der FMH-Standesordnung ist verankert, dass das Ausstellen eines Gefälligkeitszeugnisses unzulässig ist.

"Von einem Gefälligkeitszeugnis ist dann die Rede, wenn eine Ärztin bzw. ein Arzt ihren Patienten eine Arbeitsunfähigkeit bescheinigen, obschon sie oder er weiss, dass diese Arbeitsunfähigkeit nicht oder nicht im bescheinigten Umfang besteht» [10]. Im Jahre 2008 bestätigte das Bundesgericht die Verurteilung eines Arztes wegen eines falsch ausgestellten ärztlichen Zeugnisses. Das Bundesgericht präzisiert dazu: «Das Zeugnis ist unwahr, wenn es ein unzutreffendes Bild vom Gesundheitszustand des Menschen vermittelt, was auch der Fall ist, wenn wesentliche Umstände verschwiegen werden" [11]. Nachfolgend die in diesem Kontext relevante strafrechtliche Bestimmung:

StGB Art. 318 Falsches ärztliches Zeugnis

- «Ärzte, Zahnärzte, Tierärzte und Hebammen, die vorsätzlich ein unwahres Zeugnis ausstellen, das zum Gebrauche bei einer Behörde oder zur Erlangung eines unberechtigten Vorteils bestimmt, oder das geeignet ist, wichtige und berechtigte Interessen Dritter zu verletzen, werden mit Freiheitsstrafe bis zu drei Jahren oder Geldstrafe bestraft.

Hat der Täter dafür eine besondere Belohnung gefordert, angenommen oder sich versprechen lassen, so wird er mit Freiheitsstrafe bis zu drei Jahren oder Geldstrafen bestraft.

- Handelt der Täter fahrlässig, so ist die Strafe Busse.»

\section{Literatur}

1 Urteil BGer 6B_99/2008 vom 18.3.2008 E. 2.4.1.

2 Bundesamt für Gesundheit BAG $\rightarrow$ Versicherungen $\rightarrow$ Krankenversicherung $\rightarrow$ Laufende Revisionsprojekte $\rightarrow$ KVG-Änderung: Massnahmen zur Kostendämpfung - Paket 2

3 Erläuternder Bericht Massnahmenpaket Kostendämpfung 2. Bundesamt für Gesundheit BAG $\rightarrow$ Versicherungen $\rightarrow$ Krankenversicherung $\rightarrow$ Laufende Revisionsprojekte $\rightarrow$ KVG-Änderung: Massnahmen zur Kostendämpfung - Paket 2

4 Ärztliche Zeugnisse Kanton Zürich. Kanton Zürich $\rightarrow$ Handbuch Personalrecht $\rightarrow$ Krankheit und Unfall $\rightarrow$ Ärztliche Zeugnisse

5 medgate $\rightarrow$ Arbeitsunfähigkeitszeugnis

6 srf $\rightarrow$ Telemedizin im Tessin für Covid-Kranke

7 Nach Art. 14 Abs. 2bis OR ist die qualifizierte elektronische Signatur gemäss Bundesgesetz über die elektronische Signatur (ZertEs) der eigenhändigen Unterschrift gleichgestellt.

8 BGE 143 IV 209.

9 BGE 143 IV 209 E. 1.2. Der vom Arbeitgeber eingesetzte Vertrauensarzt untersteht somit dem Berufsgeheimnis nach Art. 321 StGB.

10 Ärztliche Zeugnisse Kanton Zürich. Kanton Zürich $\rightarrow$ Handbuch Personalrecht $\rightarrow$ Krankheit und Unfall $\rightarrow$ Ärztliche Zeugnisse

11 Urteil BGer 6B_99/2008 vom 18.3.2008 E.3.1.

Bildnachweis

Agenturfotografin | Dreamstime.com

\section{Das Wichtigste in Kürze}

- Dass per telemedizinische Konsultation ein Arztzeugnis ausgestellt werden kann, ist unbestritten, doch es stellt sich die Frage, welchen Beweiswert ein Arztzeugnis hat, das auf einer telemedizinischen Konsultation basiert.

- Eine eingescannte Unterschrift oder der Hinweis des Arztes, das Dokument sei elektronisch visiert worden, erfüllt die rechtliche Verbindlichkeit einer Urkunde nicht.

- Die Ärztin, die von einem Arbeitgeber beauftragte wird, die Arbeitsfähigkeit eines Arbeitnehmers zu beurteilen, untersteht dem Berufsgeheimnis gemäss Art. 321 Ziff. 1 StGB. Basierend auf der Ermächtigung des Arbeitnehmers, darf die Ärztin dem Arbeitgeber keine über den Rahmen von Art. 328b OR weitergehende Information erteilen. Das heisst, der Arbeitgeber darf über den Arbeitnehmer nur diejenigen Daten bearbeiten, soweit sie dessen Eignung für das Arbeitsverhältnis betreffen oder zur Durchführung des Arbeitsvertrages erforderlich sind. 